\title{
CF/PAni/MWNT Composites Material, A Novel Electrode to Supercapacitor
}

Dalva A. Almeida, Carla Polo Fonseca, Mauricio Ribeiro Baldan and Neidenei Gomes Ferreira

ECS Trans. 2012, Volume 41, Issue 21, Pages 13-19.

doi: 10.1149/1.3695097

Email alerting Receive free email alerts when new articles cite this article - sign up in the service box at the top right corner of the article or click here 


\title{
CF/PANI/MWNT COMPOSITES MATERIAL, A NOVEL ELECTRODE TO SUPERCAPACITOR
}

\author{
D. A. L. Almeida, C. P. Fonseca, M. R. Baldan and N. G.Ferreira. \\ Laboratório Associado de Sensores e Materiais \\ Instituto Nacional de Pesquisas Espaciais - INPE \\ Av. dos Astronautas 1758, 12245-970 - S. J. Campos, SP
}

\begin{abstract}
In this work, the binary and ternary composites composed respectively by carbon fiber/polyaniline(CF/PAni) and carbon fiber/multiwall carbon nanotubes/polyaniline (CF/PAni/MWCNT) were synthesized by oxidative polymerization. The composite materials were physically characterized by Scanning electron microscopy, by Raman spectroscopy and electrochemically by cyclic voltammetry. These hybrid composites were designed to be used as supercapacitors. By Raman spectroscopy, a higher conductivity for the CF/PAni/MWCNT composite was observed composite was observed .The highest specific capacitance was achieved to $\mathrm{CF} / \mathrm{PAni} / \mathrm{MWCNT}\left(400 \mathrm{Fg}^{-1}\right)$ which is a good candidate to the supercapacitor electrode.
\end{abstract}

\section{Introduction}

Conducting polymers represent an interesting class of electrode materials for supercapacitors, due to their high kinetics of the electrochemical charge-discharge processes. Besides, the charge is stored throughout the volume of the polymer material. Furthermore, it can be produced at a lower cost than the one for noble metal oxides. The conducting polymers can exist in either two or three general states. The polymers tend to be oxidized or 'p-doped', being positively charged with a high electronic conductivity. When the p-doped polymer is reduced, the 'undoped' state is formed. Usually, this state is insulating or semi-insulating, depending on the degree of completion of the undoping process. (1-5).

Among the conductive polymers, polyaniline (PAni) is considered the most promising electrode material for supercapacitors, because of the following characteristics: excellent capacity for energy storage, easy synthesis, high conductivity, low price of aniline monomers and its facility of manipulation. In addition, the PAni has a better accessibility to the ions for the electrochemically active surface. This can be responsible for the higher values of capacitance $(4,6)$. However, the main disadvantage of the use of the conducting polymer to make supercapacitors is its poor cycle life, due to the volume change during the doping and undoping process. Therefore, it is necessary to support adequately the electrochemical active sites of conducting polymers and this can be carried out by the addition of conducting material with a large surface area, such as carbon fiber or carbon nanotubes (CNTs). In addition, these materials present many attractive properties, such as large a surface area, high conductivity, temperature stability and percolated pore structure. Moreover, the CNT can be easily functionalized targeting different applications. $\mathrm{CNT} /$ conducting polymers have been extensively studied due to their superior performance.

Porous carbons such as carbon fibers are extremely attractive as electrode materials due to their large specific surface area, their high pore accessibility, their excellent thermal and 
chemical stability, as well as their relative low cost. The storage of electric charges in these materials are purely non-Faradaic, and the accumulation of ionic charges occurs on the double-layer at the electrode/electrolyte interface (6). The capacitive behavior of carbon materials can be further improved by the presence of active species such as conducting polymer, that contribute to the total specific capacitance (7-10).

In this work, the Polyaniline (PAni) and multi walls carbon nanotubes (MWNTs) was chemically deposited on the carbon fibers (CFs). The aim of this work is to design a hybrid composite, $\mathrm{CF} / \mathrm{PAni} / \mathrm{MWNT}$ and $\mathrm{CF} / \mathrm{PAni} /$ to be used as an electrode to the supercapacitors.

\section{Experimental Procedure}

The carbon fibers were cut in size $2 \times 1 \mathrm{~cm}$ and weighed. We used as carbon nanotube, a MWNTs with $90 \%$ purity. The MWNTs were functionalized with $\mathrm{H}_{2} \mathrm{SO}_{4}$ and $\mathrm{HNO}_{3}$ in the ratio 3:1 for 24 hours. The MWNTs functionalized were dispersed in aqueous solution containing $0.1 \mathrm{~mol} \mathrm{~L}^{-1}$ of the surfactant sodium dodecyl sulfate. This solution was added in a solution containing distilled aniline $\left(12.6 \mathrm{mmol} \mathrm{L}^{-1}\right)$ and $1.0 \mathrm{~mol} \mathrm{~L}^{-1} \mathrm{HCl}, 3.0 \mathrm{~mol} \mathrm{~L}-$ ${ }^{1} \mathrm{NaCl}$ at $-10^{\circ} \mathrm{C}$.

In a ring-shaped support, six carbon fibers $($ size $2 \times 1 \mathrm{~cm})$ were attached equally spaced and fully immersed in a monomer solution at $-10^{\circ} \mathrm{C}$. CF/PAni/NTC composites were synthesized by oxidative polymerization. The oxidizing agent solution was added in the center of the support at 90 min of the deposition time at $-10^{\circ} \mathrm{C}$ with vigorous stirring. The composite was washed with $1.0 \mathrm{~mol} \mathrm{~L}^{-1} \mathrm{HCl}$. All samples were dry in air atmosphere until a constant mass. After completely dried, the samples were weighed using an analytical balance to six decimal places.

The composite was washed with $1.0 \mathrm{~mol} \mathrm{~L}^{-1} \mathrm{HCl}$, obtaining the conducting state of the polyaniline (esmeraldine). The binary composites, CF/PAni, were produced without MWNTs with aim to observe its influence in the electrochemical properties of the electrode.

Likewise, the PAni powder was synthesized by chemical polymerization using the same solutions of composite to create the PAni electrode. The oxidizing agent solution was added during 90 min with vigorous stirring. The precipitate was collected by filtration, and then washed with $1 \mathrm{~mol} \mathrm{~L}^{-1} \mathrm{HCl}$, obtaining the conducting state of the polyaniline (emeraldine). The PAni electrode were prepared by mixing of PAni powder $(90 \% \mathrm{wt}$.) and with poly (vinylidene fluoride) PVDF (10\% wt. MW $\left.=10^{5} \mathrm{~g} \mathrm{~mol}^{-1}\right)$ in N.N.-dimethyl acetamide. The films were obtained by paint on platinum electrode. Prior to use, the electrodes were dried at room temperature for $72 \mathrm{~h}$. Morphological characterization was carried out by using scanning electron microscopy JEOL model JSM-5900LV. Raman spectra were recorded using a micro - Raman scattering spectroscopy (Renishaw microscope system 2000) with an excitation of Ar, $514.5 \mathrm{~nm}$ laser with a 500x objective.

The CF/PAni composites were electrochemically characterized by chronopotenciometry. The charge-discharge curves were obtained by applying constant $\mathrm{i}$ $\pm 1.0 \mathrm{~mA}\left(\mathrm{E}_{\text {cut-off }}=-0.1\right.$ and $\left.0.8 \mathrm{~V}\right)$. All measurements were performed in $1.0 \mathrm{~mol} / \mathrm{L} \mathrm{H}_{2} \mathrm{SO}_{4}$ solution deaered for $30 \mathrm{~min}$.

\section{Results and discussion}

SEM images in Figure 1(a-c) exhibit the CFs, PAni powder and MWCNT, respectively. The average diameter of the CF (Fig.1a) was estimated to be $9 \mu \mathrm{m}$. The polyaniline powder morphology showed the presence of fine agglomerated particles, (Fig.1b). The MWCNT 
shows an agglomeration of bunch of tubes which present a residual catalyst at the tip of MWCNT. Figure 1(d-f) shows the binary composites (CF/PAni). The images depict the PAni uniformly distributed all over the CFs. This suggests that the interaction between polymer molecules and CFs overcomes the Van der Waals interaction among the CFs. In these composites, CFs can offer a good mechanical support to the PAni and also ensure the electronic conduction in the composite electrode. In addition, clusters of PAni were observed among the carbon fibers which show an excess of the PAni due to the long deposition time.

The ternary composites CF/PAni/MWCNT in Fig. 1(g-i) present a complete coating of MWCNT over the CF/PAni substrate. For the CF/PAni/MWCNT, the PAni plays an important role as a binder between carbon fibers and MWCNT. This ternary composite shows a superficial area and roughness higher than those for CF/PAni composite.

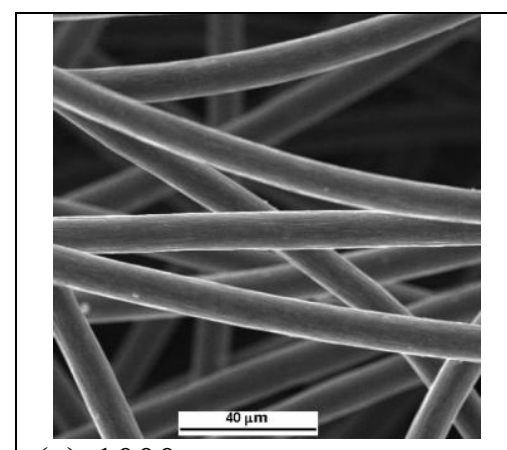

(a)-1000x
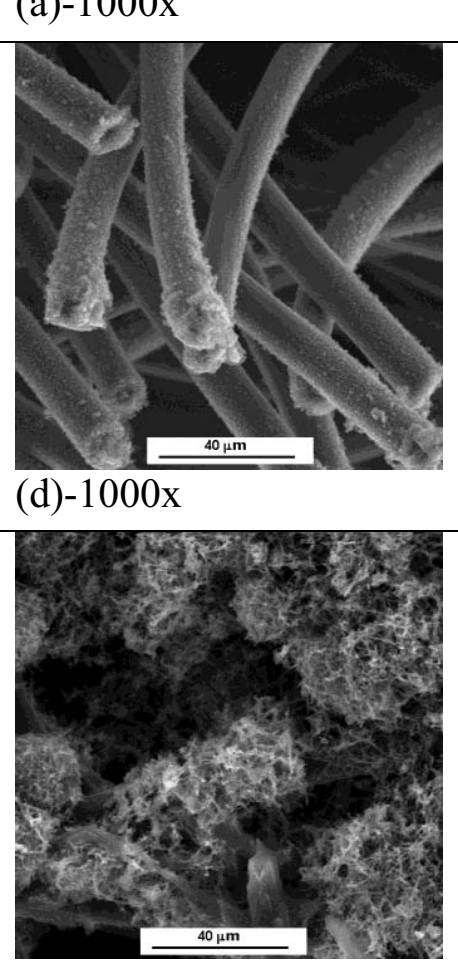

(g)-1000x

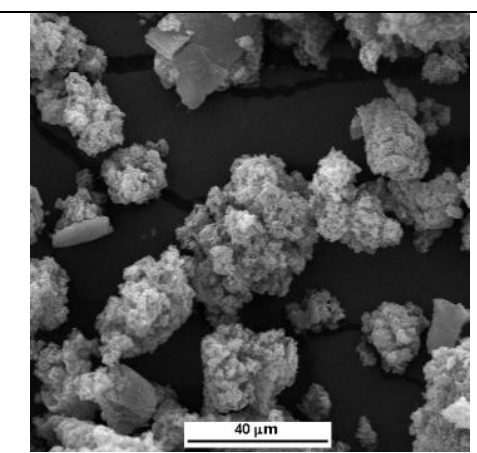

(b) $-1000 x$

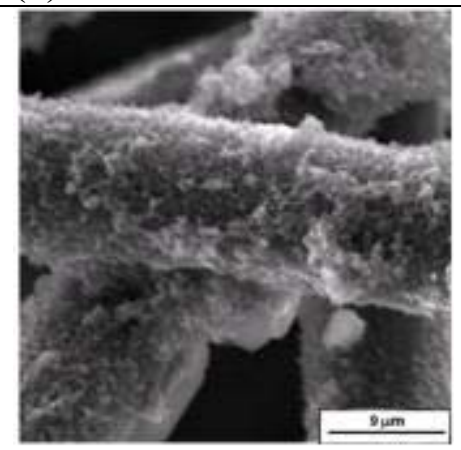

(e)-3500x

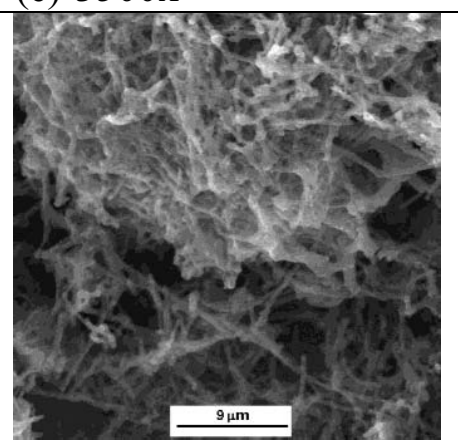

(h) $-3500 \mathrm{x}$

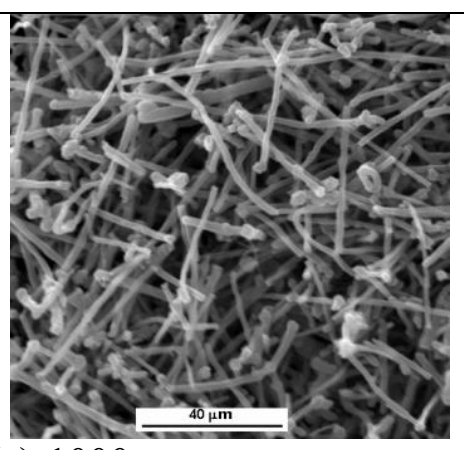

(c)-1000x

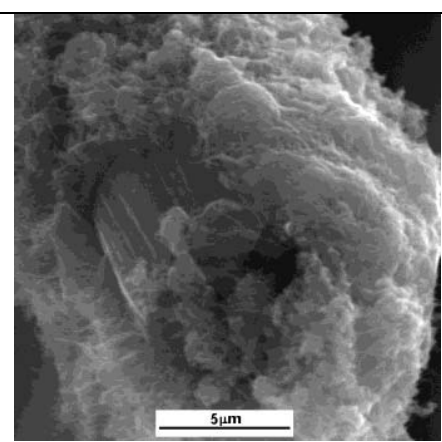

(f) $-7500 x$

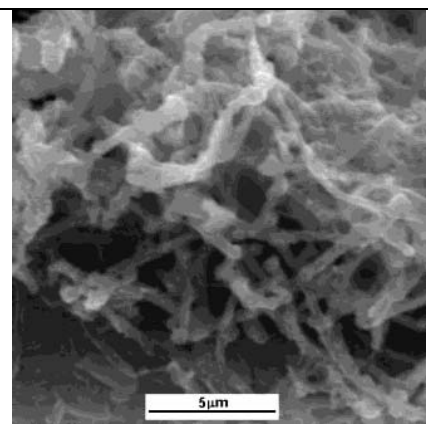

(i) $-7500 x$

Figure 1. SEM images of the (a) CFs; (b) PAni powder; (c) MWCNT. The images (d-f) $\mathrm{CF} / \mathrm{PAni}$, and the images (g-i) CF/PAni/MWCNT are presented at different magnitudes. 
Figure 2 (a-e) shows the Raman spectra of the CF, MWCNT, PAni powder, CF/PAni and $\mathrm{CF} / \mathrm{PAni} / \mathrm{MWCNT}$ composites, respectively. Both the binary and ternary composites show a spectrum dominated by the PAni, indicating a good coating of the conducting polymer on the carbon fibers. The polyaniline spectrum exhibits bands characteristic of conducting specie (Fig.2c). In the range of the 1100-1210 $\mathrm{cm}^{-1}$ the band region corresponds to $\mathrm{C}-\mathrm{H}$ in-plane bending benzene or quinone type rings, and is related to the high degree of oxidation. In the range of $1210-1520 \mathrm{~cm}^{-1}$ the band region may be assigned to $\mathrm{C}-\mathrm{N}$ and $\mathrm{C}$ $=\mathrm{N}$ stretching vibrations. By comparison, the relative intensity of the band at $\sim 1340 \mathrm{~cm}^{-1}$ is more evidenced for the ternary (Fig.2e) composite than that for the binary (Fig.2d) composite. This vibration mode is associated with the $\mathrm{C} \sim \mathrm{N}^{+}$(radical cation). This is a strong evidence that polyaniline is in the emeraldine salt polymeric form. Besides, the decrease of the band at $1480 \mathrm{~cm}^{-1}$ is an indicative of the increase in electrical conductivity of polyaniline with MWCNT (11). In addition, 1520-1650 $\mathrm{cm}^{-1}$ correspond to the $\mathrm{C}-\mathrm{C}$ and $\mathrm{C}=\mathrm{C}$ stretching vibrations of the benzene and quinoid ring, respectively. In this region, we can note the presence of the $\mathrm{C}-\mathrm{C}$ stretching band for all composites. This band may be related to benzenoid and quinoid ring.

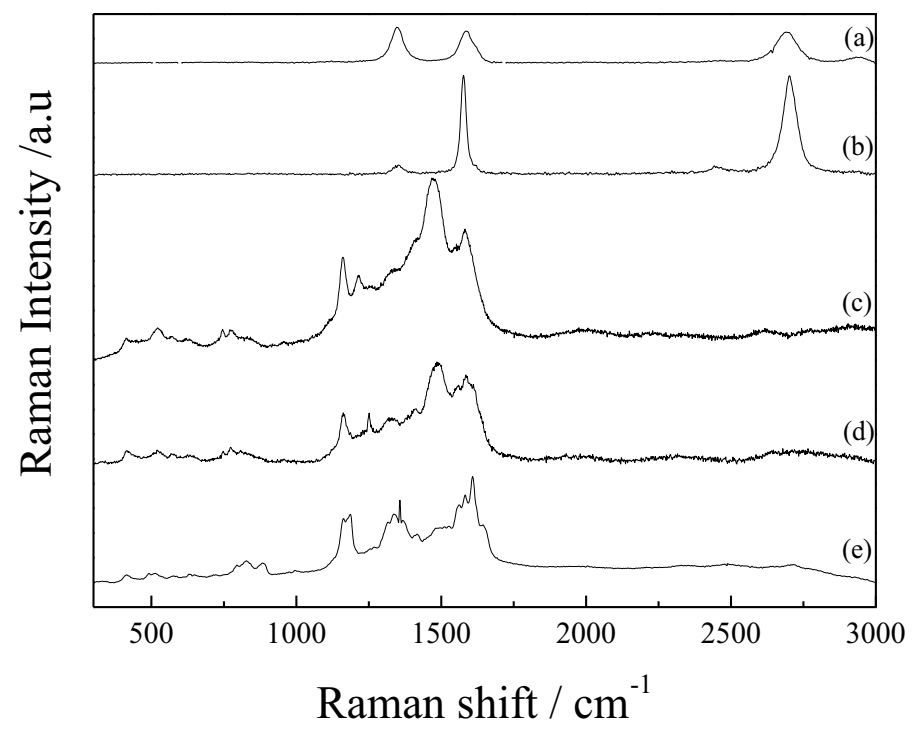

Figure 2 Raman spectrum of the (a) CF; (b) MWCNT; (c) PAni powder (d) CF/PAni; (e) CF/PAni/MWCNT.

The electrochemical performance of carbon fiber, PAni, CF/PAni and, CF/PAni/MWCNT were analyzed by cyclic voltammetry. Figure 3 shows the cyclic voltamograms of all materials, normalized by the mass of each sample. The cycle performance was not investigated over $0.78 \mathrm{~V}$ vs. $\mathrm{Ag} / \mathrm{AgCl}$ to avoid the polyaniline degradation. It can be seen that the $\mathrm{CF}$ has a purely capacitive profile assigned to the electric double layer formation. At lower potentials, the reduction process of hydrogen can be observed (inset of Fig.3).

The cyclic voltammetry of the PAni powder shows a pair of redox peaks at $0.23 \mathrm{~V}$ and other at $0.10 \mathrm{~V}$. These peaks have been assigned to the transition from leucoemeraldine state to emeraldine state (12). The second pair of redox peak of the PAni, which is related to the 
emeraldine / pernigraniline transition was not observed in cyclic voltammogram (Fig.3). The emeraldine / pernigraniline transition can not be observed because during the production process of the PAni, the mixing of PVDF with the PAni favors the formation of clusters making it difficult to observe this transition. The CF/PAni /MWCNT cyclic voltammetry shows peaks that can be attributed to the different oxidation states of the polyaniline. The redox peaks at $0.28 \mathrm{~V}$ and $0.062 \mathrm{~V}$ vs. $\mathrm{Ag} / \mathrm{AgCl}$ have been assigned to the transition from leucoemeraldine state to emeraldine state [12]. In the CF/PAni voltammograms, all the redox processes of the PAni were not clearly observed. This fact may be attributed to a disordered which may occurs during the growth process of polymer chains on the $\mathrm{CF}$.

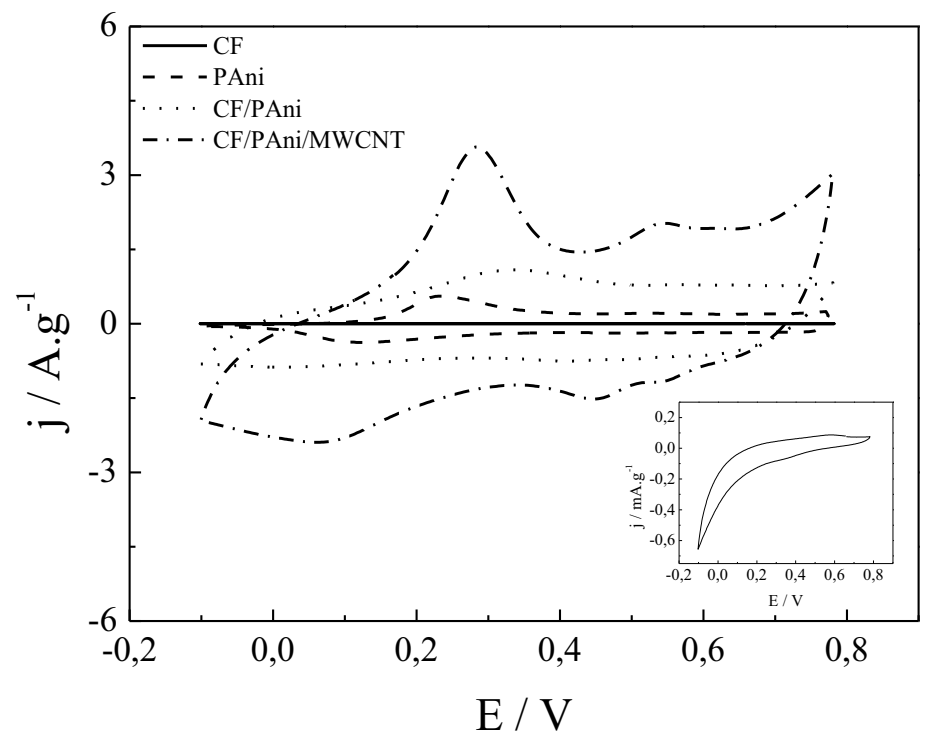

Figure 3: Cycles voltammetry of the CF, PAni, CF/PAni and CF/PAni/MWCNT electrodes, $v=10 \mathrm{mVs}^{-1}$.

The specific capacitance of the CF, Pani, CF/PAni and CF/PAni/MWCNT electrodes were obtained using galvanostatic charging-discharging cycling of the electrodes using current density of $\pm 1.0 \mathrm{~mA}$ as shown in Figure 4 . Specific capacitance values can be calculated from the following relationship:

$$
\mathrm{C}_{\mathrm{sp}}=1 /(\mathrm{m} \times(\mathrm{dV} / \mathrm{dt})
$$

where $\mathrm{dV} / \mathrm{dt}$ is obtained from the slope of the discharge curve and $m$ is the active electrode mass. The $\mathrm{CF}$ and PAni electrodes have specific capacitances of $\mathrm{C}_{\mathrm{spCF}}=4.77$ and $\mathrm{C}_{\mathrm{spPAni}}=$ $20.14 \mathrm{Fg}^{-1}$, respectively. The ternary composite of CF/PAni/MWCNT has the highest capacitance value of $\mathrm{C}_{\mathrm{sp}}=400 \mathrm{Fg}^{-1}$. 


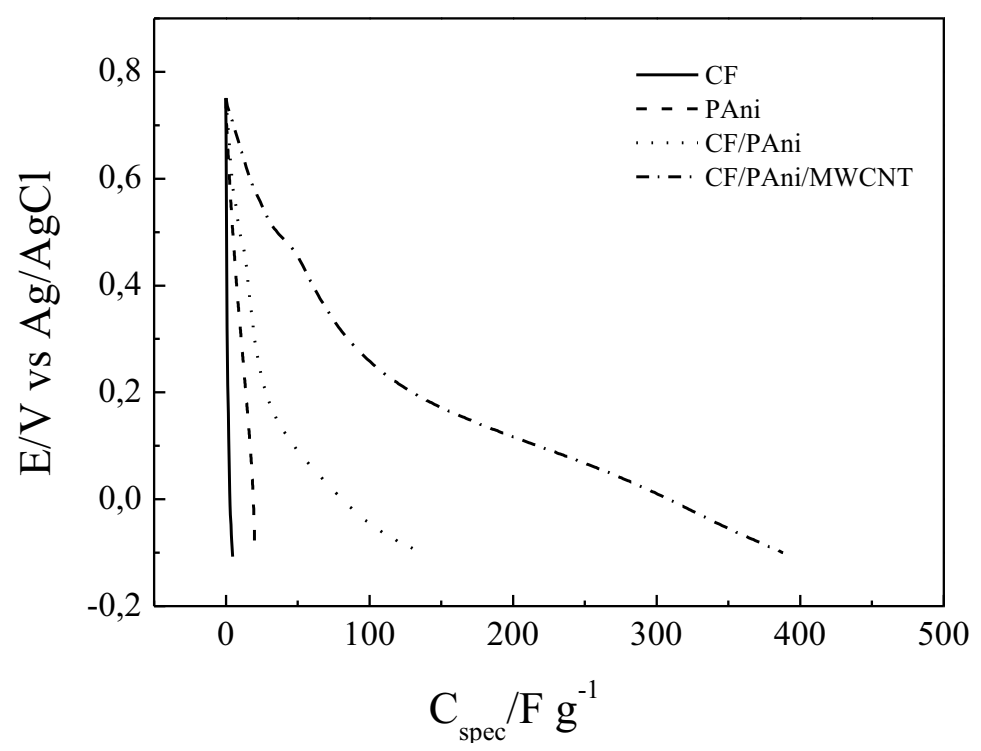

Figure 4: Specific capacitance curves of the CF, PAni, CF/PAni and CF/PAni/MWCNT with $i= \pm 1.0 \mathrm{~mA}$

\section{Conclusion}

The binary and ternary composite electrodes made of CF, MWCNT and PAni were produced using a chemical synthesis. The binary composites showed a micron thickness with polymeric clusters among the fibers. However, for the CF/PAni/MWCNT, a complete coating by MWCNT on the CF/PAni electrode showed that the PAni plays a role as binder between the $\mathrm{CF}$ and the MWCNT. Raman spectroscopy showed that the CF/PAni/MWCNT electrode was more conductive than the binary $\mathrm{CF} / \mathrm{PAni}$ electrode. This fact was evidenced by the presence of the radical cation band that was quite pronounced for this material.

From cyclic voltammetry, it was possible to verify that the highest electroactivity for the $\mathrm{CF} / \mathrm{PAni} / \mathrm{MWCNT}$ composite, due to its electrochemical reversibility. The charge/discharge cycles showed a specific capacitance at about $400 \mathrm{Fg}^{-1}$ for this ternary composite. According to the presented results, we believe that the close contact between PAni film and MWCNT and/or CF provided an increase in the electrode conductivity making the charge/discharge processes for the ternary composite faster and more effective than those for the binary composite.

\section{Acknowledgments}

This work was supported by CNPq (Process 150663/2010-2) and FAPESP (Process 2009/17584-0). 


\section{References}

1. R.B. Kaner, A.G. MacDiarmid, Synth. Met. 14, 3 (1986).

2. B. François, C. Mathis, J. Phys. 44, 21 (1983).

3. N.S. Murthy, R.H. Baughman, L.W. Shacklette, H. Fark, J. Fink, Solid State Commun. 78, 691 (1991).

4. K. Kaneto, S. Ura, K. Yoshino, Y. Unuishi, J. Appl. Phys. 23, L189 (1984).

5. K. Kaeriyama, S. Tanaka, M.-A. Sato, K. Hamada, Synth. Met. 28, C611 (1989).

6. C. Kim, Y.-O. Choi, W.-J. Lee and Kap-Seung Yang Electrochimica Acta, 50883 (2004)

7. K. Xia, Q.Gao, J. Jiang, J. Hu Carbon 461, 718 ( 2008)

8. P. Staiti, F. Lufrano Electrochimica Acta 557, 436 (2010)

9. J. Zhao, C. Lai, Y. Dai, J. Xie Materials Letters 614, 639 ( 2007)

10.Y.Tian, Y. Song, Z. Tang, Q. Guo, L. Liu Journal of Power Sources 184, 675 ( 2008)

11. E. Frackowiak, V. Khomenko, K.Jurewicz, K.Lota, F. Beguin, J. Power Sources 153 413 (2006).

[12] S.-B. Yoon, E.-H. Yoon, K.-B. Kim, Journal of Power Sources 196 (2011) 10795. 